\title{
Interdiffusion-driven synthesis of tetragonal chromium (III) oxide on $\mathrm{BaTiO}_{3}$
}

\author{
M. Asa,${ }^{1}$ G. Vinai, ${ }^{2}$ J. L. Hart, ${ }^{3}$ C. Autieri, ${ }^{4}$ C. Rinaldi, ${ }^{1}$ P. Torelli, ${ }^{2}$ G. Panaccione,${ }^{2}$ M. L. Taheri, ${ }^{3}$ \\ S. Picozzi, ${ }^{4}$ and M. Cantoni ${ }^{1, *}$ \\ ${ }^{1}$ Dipartimento di Fisica, Politecnico di Milano, Via G. Colombo 81, 20131 Milano, Italy \\ ${ }^{2}$ Laboratorio TASC, IOM-CNR, S.S. 14 km 163.5, Basovizza, I-34149 Trieste, Italy \\ ${ }^{3}$ Department of Materials Science and Engineering, Drexel University, 3141 Chestnut Street, Philadelphia, Pennsylvania 19104, USA \\ ${ }^{4}$ Consiglio Nazionale delle Ricerche CNR-SPIN, UOS L'Aquila, Sede Temporanea di Chieti, I-66100 Chieti, Italy
}

(Received 4 December 2017; published 9 March 2018)

\begin{abstract}
Interfaces play a crucial role in the study of novel phenomena emerging at heterostructures comprising metals and functional oxides. For this reason, attention should be paid to the interface chemistry, which can favor the interdiffusion of atomic species and, under certain conditions, lead to the formation of radically different compounds with respect to the original constituents. In this work, we consider $\mathrm{Cr} / \mathrm{BaTiO}$ heterostructures grown on $\mathrm{SrTiO}_{3}$ (100) substrates. Chromium thin films (1-2 nm thickness) are deposited by molecular beam epitaxy on the $\mathrm{BaTiO}_{3}$ layer, and subsequently annealed in vacuum at temperatures ranging from 473 to $773 \mathrm{~K}$. A disordered metallic layer is detected for annealing temperatures up to $573 \mathrm{~K}$, whereas, at higher temperatures, we observe a progressive oxidation of chromium, which we relate to the thermally activated migration of oxygen from the substrate. The chromium oxidation state is +3 and the film shows a defective rocksalt structure, which grows lattice matched on the underlying $\mathrm{BaTiO}_{3}$ layer. One out of every three atoms of chromium is missing, producing an uncommon tetragonal phase with $\mathrm{Cr}_{2} \mathrm{O}_{3}$ stoichiometry. Despite the structural difference with respect to the ordinary corundum $\alpha-\mathrm{Cr}_{2} \mathrm{O}_{3}$ phase, we demonstrate both experimentally and theoretically that the electronic properties of the two phases are, to a large extent, equivalent.
\end{abstract}

DOI: 10.1103/PhysRevMaterials.2.033401

\section{INTRODUCTION}

Interfacing metallic and oxide thin films provides both a large variety of applications and a series of technological challenges. In fact, the possibility of combining metal oxides with metallic layers is fundamental not only for microelectronics, but also to provide a testbed for new physical phenomena, which may arise from the combination of functional oxides and metals. It is very well known, however, that the interfaces between reactive metals and oxides may become unstable, especially when exposed to high thermal load, as diffusion of chemical species across the interface follows an Arrheniustype relation. Interestingly, interdiffusion can also lead to the formation of new interfacial phases, such as transition-metal oxides, which may exhibit novel properties [1-3].

In this work, we focus on the $\mathrm{Cr} / \mathrm{BaTiO}_{3}$ system. $\mathrm{BaTiO}_{3}$ (BTO) is a prototypical ferroelectric oxide with perovskite structure, widely employed for different applications, from magnetoelectric coupling with metals $[1,4,5]$ and oxides [6], to electroresistive devices [7] even with memristive capabilities [8], to dedicated applications exploiting its piezoelectric, pyroelectric, and/or electro-optic properties [9]. Chromium is a highly reactive $3 d$ metal, relatively abundant on earth [10], often used as an adhesion layer between noble metals and oxides for the realization of electrical contacts in microfabricated devices. Moreover, it is antiferromagnetic at room temperature

\footnotetext{
*Author to whom correspondence should be addressed: matteo.cantoni@polimi.it
}

[11], with a bulk Néel temperature of $311 \mathrm{~K}$, and can be epitaxially grown on different substrates, both metals (e.g., $\mathrm{Au}$ [12], $\mathrm{Ag}$ [13], and Fe [14]) and oxides (e.g., $\mathrm{MgO}$ [15], $\mathrm{SrTiO}_{3}$ [16], $\mathrm{TiO}_{2}$ [17], and $\mathrm{Al}_{2} \mathrm{O}_{3}$ [18]). Because of the high chemical reactivity, oxide underlayers can lead to $\mathrm{Cr}$ oxidation under particular growth conditions. For example, Ref. [16] reports a detailed study of $\mathrm{Cr}$ growth by electron beam evaporation on $\mathrm{SrTiO}_{3}$ (STO) substrates. Reference [19] demonstrates the oxygen diffusion from STO across the interface when the film is heated above $900 \mathrm{~K}$ and reports the formation of a chromium oxide layer whose chemical composition and crystallographic structure could not be detected unequivocally. The temperature at which this solid-state reaction begins was found to be strongly dependent on the electronic structure of the oxide itself [20]. For this reason, given the chemical and structural similarities between BTO and STO, it is interesting to understand what happens at the $\mathrm{Cr} / \mathrm{BTO}$ interface and to determine the conditions for which the interface between these two materials is stable.

To this end, we performed a systematic study of Cr ultrathin (1-2 nm) films, grown by molecular beam epitaxy and followed by annealing at different temperatures, on $\mathrm{BaTiO}_{3}$ (001) underlayers. Using X-ray photoelectron spectroscopy (XPS), we verified that a metallic $\mathrm{Cr}$ interface is maintained for annealing temperatures up to $573 \mathrm{~K}$, whereas higher temperatures lead to the oxidation of the $\mathrm{Cr}$ layer. By combining $\mathrm{x}$-ray photoelectron diffraction (XPD), x-ray absorption spectroscopy (XAS), low-energy electron diffraction (LEED), reflection high-energy electron diffraction (RHEED), transmission electron microscopy (TEM), scanning TEM (STEM), 
electron energy-loss spectroscopy (EELS), and density functional theory (DFT), we verified that the chromium oxide resulting from oxygen interdiffusion represents an uncommon tetragonal phase of $\mathrm{Cr}_{2} \mathrm{O}_{3}$, which gets stabilized by the epitaxy on BTO. We also discuss how, despite the different structural phase, the chemical and electronic properties of tetragonal chromium oxide are similar to those of bulk $\mathrm{Cr}_{2} \mathrm{O}_{3}$.

\section{EXPERIMENTAL SETUP AND COMPUTATIONAL DETAILS}

The growth of $\mathrm{Cr} / \mathrm{BTO}$ heterostructures was performed by means of pulsed laser deposition (PLD) and molecular beam epitaxy (MBE) in a cluster tool where both techniques, as well as characterization tools (XPS, XPD, LEED, and RHEED), are available in situ [21].

Thirty-nm-thick BTO films were grown on commercial $\mathrm{SrTiO}_{3}$ (001) substrates by PLD in a dedicated chamber. The whole process was carried out in an oxygen atmosphere of $53.3 \mu$ bar. Before the growth, annealing of the substrate at $1000 \mathrm{~K}$ for 30 minutes was performed in order to clean and order the surface. Following a growth procedure previously optimized to realize epitaxial ferroelectric thin films [22], a quadrupled Q-switched Nd:YAG laser $(266 \mathrm{~nm})$, providing 7 -ns-long pulses with a fluence of $5.6 \mathrm{~J} / \mathrm{cm}^{2}$, has been operated at a repetition frequency of $2 \mathrm{~Hz}$ to generate a plasma from a stoichiometric target. The substrate temperature was kept at $873 \mathrm{~K}$ during the deposition. The growth was followed by an annealing in 0.5 bar of oxygen at $873 \mathrm{~K}$. Chromium layers with 1 or $2 \mathrm{~nm}$ thicknesses were deposited by MBE in ultrahigh vacuum (UHV) conditions (chamber pressure $<10^{-9}$ mbar). During the process, the substrate was maintained at room temperature and the deposition rate was set to about $1 \AA / \mathrm{min}$, as calibrated with a quartz microbalance, and later confirmed by XPS analysis. Subsequently, $\mathrm{Cr}$ films were annealed in vacuum at temperatures ranging from 373 to $773 \mathrm{~K}$ for 20 minutes.

The chemical and structural properties have been investigated in situ by XPS and XPD as a function of the annealing temperature. Photoelectrons were excited by standard Al$\mathrm{K} \alpha$ x-ray source and collected by a hemispherical energy analyzer (HEA), Phoibos 150 (SPECS GmbH), yielding an acceptance angle of $\pm 2.5^{\circ}$, a field view of $\sim 1.4 \mathrm{~mm}^{2}$, and an energy resolution of $1.03 \mathrm{eV}$. Due to the lack of any carbon contamination on the samples, the $\mathrm{O} 1 s$ peak of BTO has been used instead as the reference at a binding energy of $530.1 \mathrm{eV}$ [23,24].

Ex situ characterization has been carried out on a 2-nm-thick chromium oxide on BTO. The chemical stability of this oxide in ambient conditions, required for ex situ studies, has been confirmed by repeating XPS measurement after prolonged air exposure of the sample, verifying a consistent overlap between photoemission spectra (data not shown).

The oxidation state of the 2-nm-thick Cr layer annealed at $773 \mathrm{~K}$ has been determined by XAS at the APE-HE beam line of the ELETTRA synchrotron facility [25] by measuring the total electron yield (TEY) at $\mathrm{Cr} L_{23}$ and $\mathrm{O} K$ edges at room temperature.

This sample was also studied with a JEOL 2100F (Schottky source) transmission electron microscope (TEM). The sample was prepared for TEM via a standard focused ion beam (FIB) lift-out procedure, with final FIB thinning performed with $5 \mathrm{keV}$ Ga ions and subsequent ion milling with $3 \mathrm{keV}$ Ar ions. The structure of the $\mathrm{Cr} / \mathrm{BTO}$ interface was investigated with high-resolution (HR) TEM, whereas the oxidation state of the $\mathrm{Cr}$ and BTO films was studied with STEM and direct-detection (DD) EELS [26]. For EELS, a dispersion of $0.125 \mathrm{eV} /$ channel was used with a collection semiangle of $30 \mathrm{mrad}$.

First-principles density functional theory calculations were performed within the generalized gradient approximation (GGA) by using the plane-wave VASP [27] package. We adopted the PBEsol for the exchange-correlation functional [28] to determine the unknown internal coordinates, as it was shown to give excellent results for transition-metal oxides. The Hubbard $U$ effects on the $\mathrm{Cr}$ sites were included within the GGA $+U$ [29] approach using the rotational invariant scheme [30]. Using a value of the Hund coupling constant $J_{\mathrm{H}}=0.15 U$, we performed the calculations for $U=0,3$, and $6 \mathrm{eV}$, where the latter value is generally used for ionic $\mathrm{Cr}$ compounds. The core and the valence electrons were treated with the projector augmented wave method [31] and an energy cutoff of $400 \mathrm{eV}$ for the plane-wave basis was used. We assumed the experimental crystal symmetry for $\mathrm{Cr}_{2} \mathrm{O}_{3}$ in bulk form, neglecting the influence of the BTO interface. To reproduce the defective rocksalt structure, we used a supercell where the lattice constants of the supercell are $a_{\text {sup }}=a / \sqrt{2}$, $b_{\text {sup }}=3 a_{\text {sup }}$, and $c_{\text {sup }}=c$. We fixed the lattice parameters to the experimental values and performed the relaxation of the internal degrees of freedom by minimizing the total-energy difference to less than $10^{-5} \mathrm{eV}$ and the remaining forces to less than $5 \mathrm{meV} / \AA$. In this symmetry, the $\mathrm{Cr}$ atoms are equivalent, while there are two types of oxygens. A $12 \times 4 \times 6 k$-point Monkhorst-Pack grid [32] was used for the Brillouin zone sampling.

\section{RESULTS}

\section{A. Chemical properties}

\section{Thermal stability}

In order to evaluate the chemical stability of $\mathrm{Cr}$ thin films on BTO, XPS has been performed on as-deposited and annealed samples. Figure 1(a) presents the $\mathrm{Cr} 2 p$ peak measured for different annealing temperatures on a $\mathrm{Cr}(1 \mathrm{~nm}) / \mathrm{BTO}(30 \mathrm{~nm})$ bilayer. In the as-grown sample, (i) both the binding energy $(\mathrm{BE}=574.35 \pm 0.15 \mathrm{eV})$ and the shape of the $\mathrm{Cr} 2 p_{3 / 2}$ peak point towards a zero-oxidation state. After annealing at $473 \mathrm{~K}$ (ii) and $573 \mathrm{~K}$ (iii), no evident modifications are found, thus indicating that the metallic character of $\mathrm{Cr}$ is preserved.

In contrast, following the annealing at $673 \mathrm{~K}$ (iv), the peak position slightly moves towards higher BE and a shoulder appears on its left side. These features can be ascribed to the appearance of an oxide component in the $\mathrm{Cr}$ film. Finally, the annealing carried out at $773 \mathrm{~K}$ (v) results in the almost complete oxidation of the chromium layer, as can be inferred from the radical change in the peak shape and the shift of its maximum to definitely higher $\mathrm{BE}(576.7 \pm 0.15 \mathrm{eV})$. The peak is fitted by two doublets corresponding to metallic [dashed line in Fig. 1(a)] and oxidized [dotted line in Fig. 1(a)] components, after subtraction of a Shirley background due 

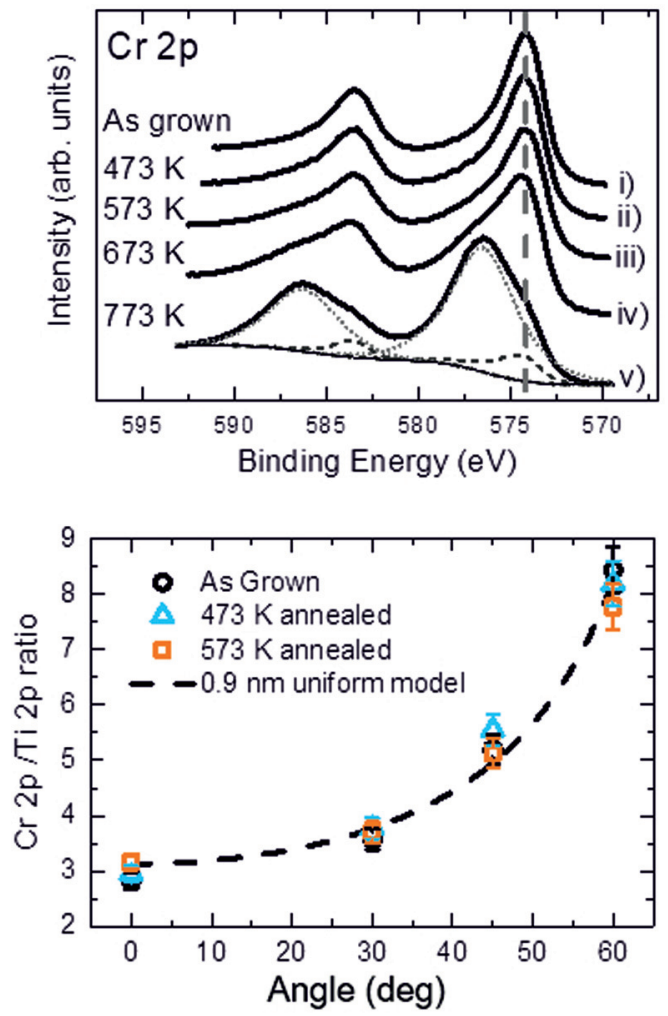

(b)

FIG. 1. (a) Cr $2 p$ spectra as measured by XPS as a function of the annealing temperature. The peak is fitted by two doublets corresponding to metallic (dashed line) and oxidized (dotted line) components. (b) $\mathrm{Cr} 2 p / \mathrm{Ti} 2 p$ ratio for as grown and annealed samples ( 473 and $573 \mathrm{~K}$ ) as a function of the take-off angle. The dashed line represents the data fit by Eq. (1).

to inelastic electron scattering. The metallic content is about $13 \%$, evaluated as the ratio between the areas of the metallic components and of the whole structure (metallic + oxide). This can account for disordered $\mathrm{Cr}$ atoms not embedded in the Cr oxide structure, but it does not affect the following analysis.

We note that the progressive oxidation of the Cr layer on BTO is obtained by annealing the samples at a temperature significantly lower with respect to the case of an undoped STO (where the oxidation process begins only above $900 \mathrm{~K}$ ) [17]. This difference should not be ascribed to the possible presence of crystal defects in the BTO film, as defects purposely introduced in STO do not affect the interface stability [20]. Rather, the moderate thermal load required for the oxidation of a $\mathrm{Cr}$ overlayer on BTO should be regarded as an intrinsic property of this system.

The ratio between the intensities of $\mathrm{Cr} 2 p$ and Ti $2 p$ peaks and its angular dependence has also been verified. As shown in Fig. 1(b), annealing up to $573 \mathrm{~K}$ does not produce any sensible change, ruling out any significant interdiffusion of the metallic species across the interface. Data for annealing above $573 \mathrm{~K}$ are not shown since the modulation of $\mathrm{Cr} 2 p$ intensity due to photoelectron diffraction (see Sec. IIIB) heavily affects the angular dependence of the $\mathrm{Cr} / \mathrm{Ti}$ ratio.

The data shown in Fig. 1(b) can be fitted by Eq. (1) [33], representing the ratio between $\mathrm{Cr}$ and $\mathrm{Ti}$ intensities measured on the $\mathrm{Cr} / \mathrm{BaTiO}_{3}$ bilayer. $\mathrm{Cr}$ is assumed to have a finite and
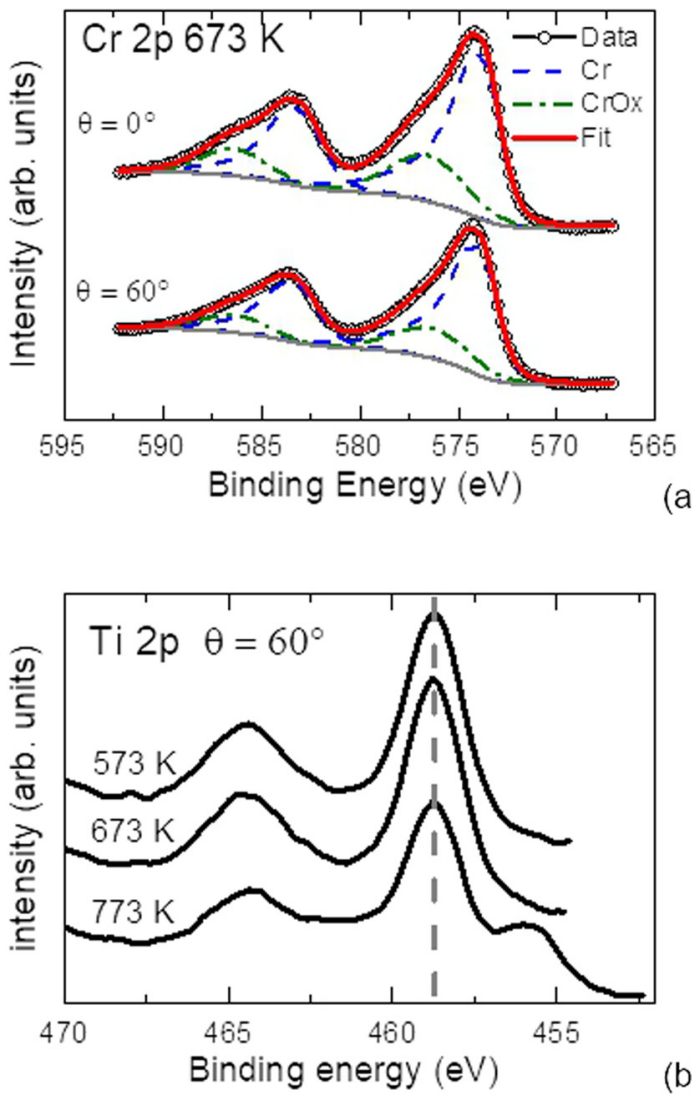

(b)

FIG. 2. (a) Comparison of normalized $\mathrm{Cr} 2 p$ peak measured at normal $\left(\theta=0^{\circ}\right)$ and grazing $\left(\theta=60^{\circ}\right)$ incidence after the annealing at $673 \mathrm{~K}$. The peak is fitted by two doublets corresponding to metallic (blue dashed line) and oxidized (green dash-dotted line) components. (b) Ti $2 p$ spectra at grazing incidence (take-off angle $\theta=60^{\circ}$ ) following the annealing at 573,673 , and $773 \mathrm{~K}$.

uniform thickness $\left(d_{\mathrm{Cr}}\right)$, whereas $\mathrm{BaTiO}_{3}(\mathrm{BTO})$ is taken as semi-infinite:

$$
\frac{I_{\mathrm{Cr}}}{I_{\mathrm{Ti}}}=\frac{N_{\mathrm{Cr}}}{N_{\mathrm{Ti}}} \frac{\lambda_{\mathrm{Cr}}}{\lambda_{\mathrm{Ti}(\mathrm{BTO})}} \frac{1-\exp \left(-d_{\mathrm{Cr}} / \lambda_{\mathrm{Cr}} \cos \theta\right)}{\exp \left(d_{\mathrm{Cr}} / \lambda_{\mathrm{Ti}(\mathrm{Cr})} \cos \theta\right)} .
$$

$I_{\mathrm{Cr}}$ and $I_{\mathrm{Ti}}$ are the $\mathrm{Cr} 2 p_{3 / 2}$ and $\mathrm{Ti} 2 p$ intensities, as measured by XPS; $N_{\mathrm{Cr}}$ and $N_{\mathrm{Ti}}$ are the $\mathrm{Cr}$ and $\mathrm{Ti}$ atomic densities; $\lambda_{\mathrm{Cr}}$ is the $\mathrm{Cr} 2 p_{3 / 2}$ electron escape depth $(1.54 \mathrm{~nm})$; $\lambda_{\mathrm{Ti}(\mathrm{BTO})}$ and $\lambda_{\mathrm{Ti}(\mathrm{Cr})}$ are the Ti $2 p$ electron escape depths in BTO $(1.94 \mathrm{~nm})$ and $\mathrm{Cr}(1.73 \mathrm{~nm})$. The escape depths were calculated with the Tanuma-Powell-Penn formula [34]. The $\mathrm{Cr}$ thickness result is $0.9 \mathrm{~nm}$, coherent with the nominal thickness $(1.0 \pm 0.1 \mathrm{~nm})$ calibrated by the quartz microbalance. The good agreement of the angular dependence of the $\mathrm{Cr} / \mathrm{Ti}$ ratio with the uniform layer model indicates a mostly planar growth of the Cr layer.

To verify whether the oxidation process occurring for temperatures above $573 \mathrm{~K}$ is related to the thermally activated migration of oxygen from the BTO or to residual gases in the UHV chamber, we performed angle-resolved measurements. The normalized spectra collected at $0^{\circ}$ and $60^{\circ}$ take-off angles after the annealing at $673 \mathrm{~K}$ are presented in Fig. 2(a). The peaks are fitted by two doublets corresponding to the metallic and oxidized components, after subtraction of a Shirley 
background due to inelastic electron scattering, as above [see Fig. 1(a)]. The line shape of as-grown $\mathrm{Cr}$ has been used to fit the metallic peak, whereas a Gaussian-Lorentzian line shape has been used for the oxide components. Due to the reduced thickness of the film $(1 \mathrm{~nm})$ with respect to the electron escape depth (1.54 nm [34]) of photoelectrons coming from the $\mathrm{Cr}$ $2 p$ peak, XPS is sensible to the whole film, with grazing incidence $\left(\theta=60^{\circ}\right)$ offering increased surface sensitivity and normal incidence $\left(\theta=0^{\circ}\right)$ containing more signal from the BTO interface.

Looking at Fig. 2(a), the oxide component (green dashdotted line) is observed in both spectra, but is relatively larger in the spectrum taken at normal incidence. The reduction of the oxide doublet at grazing incidence thus excludes a surface oxidation related to oxygen residuals possibly present in the UHV chamber, and suggests instead a migration of oxygen from the underlying BTO whose increased mobility at $673 \mathrm{~K}$ promotes interdiffusion. Using the simple model for the expected relative intensities of the oxide and metallic components in XPS spectra reported in Ref. [33] by some of the authors, we can estimate a value of $3.3 \pm 0.4 \AA$ for the thickness of the $\mathrm{Cr}$ oxide layer at the BTO interface, in the hypothesis of layers of uniform thickness.

Consistent with our picture of oxygen migrating from BTO, we also observe that as the $\mathrm{Cr}$ layer gets oxidized following the annealing at $773 \mathrm{~K}$, the Ti $2 p$ spectrum changes from a single doublet corresponding to only $\mathrm{Ti}^{4+}$ states to a broader structure indicating the presence of $\mathrm{Ti}^{3+}$ states. Figure 2(b) shows the evolution of the Ti $2 p$ XPS spectra after the annealing at 573,673 , and $773 \mathrm{~K}$. The spectra taken at grazing incidence (take-off angle $\theta=60^{\circ}$ ) are reported as they have the largest sensitivity to the BTO interface. Even though partial oxidation of chromium is already observed after the annealing at $673 \mathrm{~K}$, no clear difference is found in the Ti $2 p$ line shape. The situation is radically different in the case of the annealing at $773 \mathrm{~K}$. In this case, part of the titanium is reduced as made evident by the broadening of the structure with an additional peak at lower binding energies.

\section{Oxidation state}

To identify the chromium oxidation state, in situ XPS and ex situ XAS have been performed on the $\mathrm{Cr}$ film annealed at $773 \mathrm{~K}$. From XPS [see Fig. 1(a), bottom curve], a +3 oxidation state can be inferred, with the experimental chemical shift of the $\mathrm{Cr} 2 p_{3 / 2}$ peak $(2.27 \pm 0.18 \mathrm{eV})$ consistent with the tabulated one for $\mathrm{Cr}_{2} \mathrm{O}_{3}(2.30 \pm 0.42 \mathrm{eV})$ [35]. In the same way, the measured spin-orbit splitting $\Delta E=9.8 \pm 0.1 \mathrm{eV}$ matches the one of the reference $\mathrm{Cr}_{2} \mathrm{O}_{3}$ [36]. This is not unexpected because $\mathrm{Cr}_{2} \mathrm{O}_{3}$ presents the lowest formation enthalpy $(-1139.7 \mathrm{~kJ} / \mathrm{mol})$ with respect to other $\mathrm{Cr}$ oxides $\left(-286.8 \mathrm{~kJ} / \mathrm{mol}\right.$ for $\mathrm{CrO}_{3},-81.3 \mathrm{~kJ} / \mathrm{mol}$ for $\mathrm{CrO}_{2}$, and 198.3 $\mathrm{kJ} / \mathrm{mol}$ for $\mathrm{CrO}$ ) [37].

XAS, performed at the $\mathrm{Cr} L_{23}$ and $\mathrm{O} K$ edges, confirms this result. Figure 3(a) shows the XAS spectra at the $\mathrm{Cr} L_{23}$ edge measured on the 2-nm-thick $\mathrm{Cr}$ film annealed at $773 \mathrm{~K}$ and on the reference $\alpha-\mathrm{Cr}_{2} \mathrm{O}_{3}$ [38], $\mathrm{CrO}_{2}$ [39], $\mathrm{CrO}_{3}$ [40], and $\mathrm{CrO}$ [41] samples. The striking similarity between the XAS of $\mathrm{Cr}_{2} \mathrm{O}_{3}$ and our experimental data confirms the XPS prediction of a $+3 \mathrm{Cr}$ oxidation state. The other possible oxidation states,

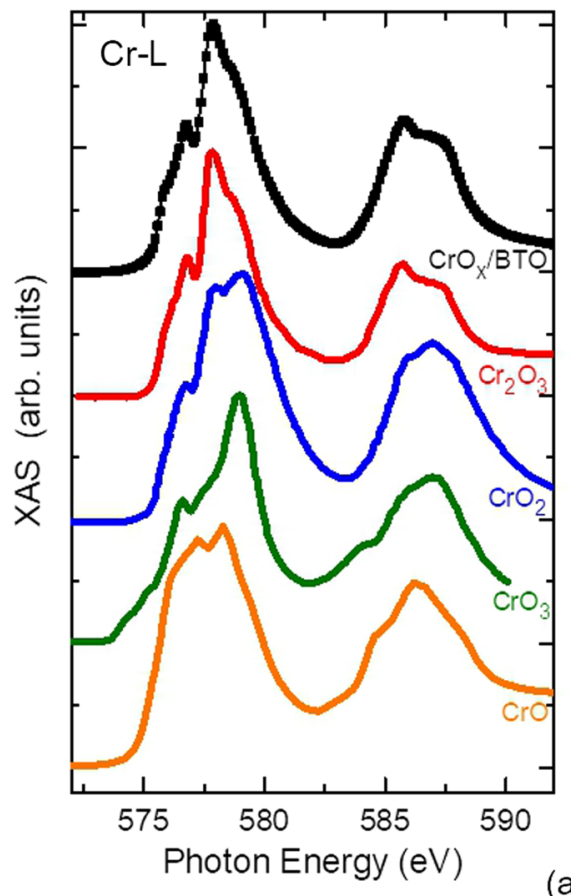

(a)

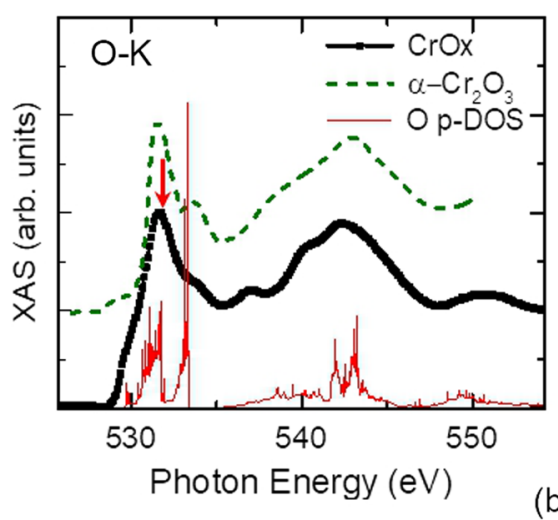

(b)

FIG. 3. (a) XAS at the $\mathrm{Cr} L$ edge of $\mathrm{Cr}$ oxide on BTO (black curve) and reference spectra for $\alpha-\mathrm{Cr}_{2} \mathrm{O}_{3}$ [38], $\mathrm{CrO}_{2}$ [39], $\mathrm{CrO}_{3}$ [40], and $\mathrm{CrO}$ [41]. (b) XAS at the $\mathrm{O} K$ edge of Cr oxide on BTO (black curve) and reference $\alpha-\mathrm{Cr}_{2} \mathrm{O}_{3}$ (green dashed curve) [43]. In red, the oxygen $p$-projected density of states (DOS) calculated from DFT on a defective rocksalt $\mathrm{Cr}_{2} \mathrm{O}_{3}$ structure is superimposed to match the experimental peaks.

+2 (as in $\mathrm{CrO}),+4\left(\mathrm{CrO}_{2}\right)$, and $+6\left(\mathrm{CrO}_{3}\right)$, can be ruled out because the corresponding XAS spectra are definitely different. The same indication has been found comparing the experimental $\mathrm{O} K$ edge, highly sensitive to the chemical environment [42], of the 2-nm-thick $\mathrm{Cr}$ film annealed at $773 \mathrm{~K}$ and of bulk $\alpha-\mathrm{Cr}_{2} \mathrm{O}_{3}$ [43] [Fig. 3(b)]. In this case, we cannot rule out the spurious contribution to the signal from oxygen in BTO due to the reduced thickness of our $\mathrm{Cr}$ oxide layer (from now on, simply called $\mathrm{CrOx}$ ). Nevertheless, the largest part of the total electron yield signal comes from the surface (i.e., from $\mathrm{CrOx}$ ) and a good qualitative agreement between the two curves is found. As we will discuss in the following sections, even if the structure of $\mathrm{CrOx} / \mathrm{BTO}$ differs from the $\alpha$ phase of $\mathrm{Cr}_{2} \mathrm{O}_{3}$, XAS and DFT show that the stoichiometry 

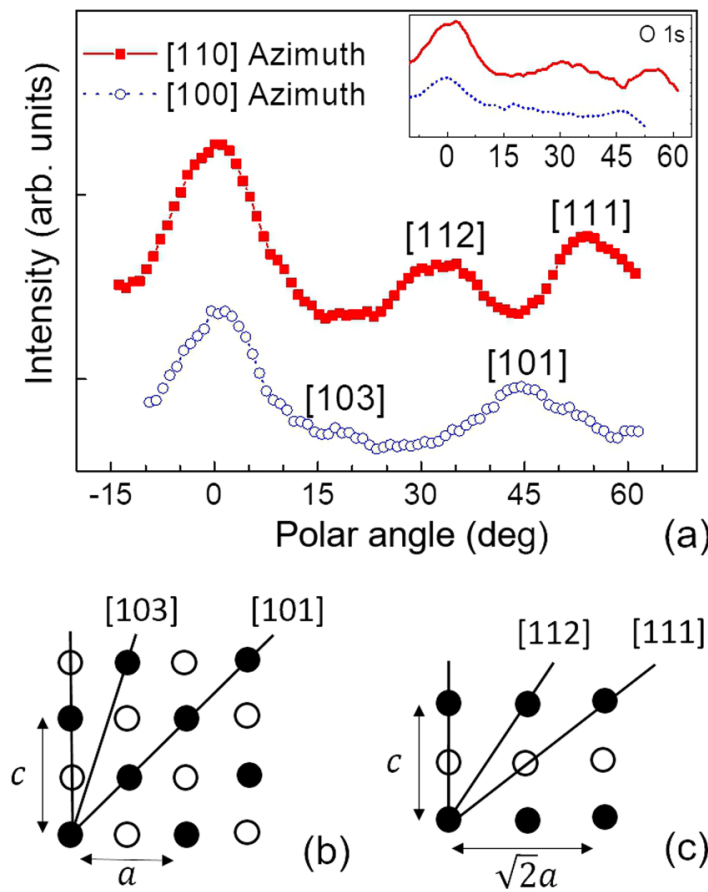

FIG. 4. (a) XPD curves of $\mathrm{Cr} 2 p_{3 / 2}$, with $1 \mathrm{~nm}$ nominal thickness and after annealing at $773 \mathrm{~K}$, along the [100] (blue empty dots, bottom) and [110] (red dots, top) azimuths of the substrate; in the inset, XPD curves of $\mathrm{O} 1 s$, with the same azimuth of $\mathrm{Cr} 2 p_{3 / 2}$, are reported. (b),(c) Atomic arrangement in the (010) and (110) planes of a rocksalt structure.

and the electronic properties are largely comparable between these two $\mathrm{Cr}$ oxides.

\section{B. Structural characterization}

The structure of the $\mathrm{Cr}$ layer has been monitored after each annealing step by XPD. The $\mathrm{Cr} 2 p_{3 / 2}$ and $\mathrm{O} 1 s$ peak intensities were recorded along the [100] and [110] azimuths of the BTO layer. Up to annealing at $573 \mathrm{~K}$, no trace of crystal order has been found. In this annealing regime, $\mathrm{Cr}$ is metallic without any oxidation shoulder, as shown by Fig. 1(a): this means that with the $\mathrm{Cr}$ preparation procedure we adopted, it is not possible to obtain ordered $\mathrm{Cr}$ films with metallic character on BTO underlayers.

Conversely, on oxidized films, a clear diffraction signal can be observed due to the appearance of a crystal order. Figure 4(a) shows the XPD signal for $\mathrm{Cr} 2 p_{3 / 2}$, measured on a $773 \mathrm{~K}$ annealed sample with $1 \mathrm{~nm} \mathrm{Cr}$ nominal thickness, along the [100] (blue empty dots) and [110] (red full dots) azimuths of the STO substrate. Note that BTO grows cube on cube on STO, so that BTO[100]//STO[100] and BTO[110]//STO[110].

Figures 4(b) and 4(c) represent the atomic arrangement in the $(010)$ and $(1 \overline{1} 0)$ planes of a rocksalt structure, respectively. The main forward scattering directions for each atomic species [e.g., the black dots in Figs. 4(b) and 4(c)] are reported: [103] and [101] in the (010) plane, [112] and [111] in the (1 $\overline{1} 0)$ plane. The corresponding diffraction angles (i.e., the angles between the scattering directions and the surface normal) are only slightly overestimated (within 3\%) with respect to the positions of the experimental peaks in Fig. 4(a). This implies a quite good crystal order because peaks are clearly emerging from the background, with a small in-plane compression of the rocksalt cell $(c / a>1)$ (see Sec. S1 of the Supplemental Material for the comparison between experimental and calculated diffraction angles [44]).

The positions and the relative intensities of the XPD peaks are compatible with a cubic lattice, with the same orientation of the substrate. The epitaxial orientation relationships thus are $\mathrm{CrOx}[100] / / \mathrm{BTO}[100] / / \mathrm{STO}[100]$ and CrOx[110]//BTO[110]//STO[110]. Moreover, the presence of the peaks corresponding to directions [112] and [103] along the [110] and [100] azimuth, respectively, reveals a face-centered structure. In fact, these directions present a larger atomic density in an FCC lattice [see, for instance, black dots in Figs. 4(b) and 4(c)], whereas a single cubic structure would present a dominant peak along [102] over [103], and a bodycentered structure would also present a dominant peak along [113] over [112]. An analogous behavior is also found when considering the $\mathrm{O} 1 s$ peak, as shown in the inset, indicating that a similar FCC coordination is present in the oxygen atoms as well. In the partially oxidized film annealed at $673 \mathrm{~K}$, the XPD signal is fundamentally the same as the $773 \mathrm{~K}$ annealed film, with only minor differences. In all cases, the XPD features come from the oxidized part of the $\mathrm{Cr}$ film only. In fact, XPD indicates a cube-on-cube growth, whereas in the case of metallic $\mathrm{Cr}$, a $45^{\circ}$ rotation will be expected in order to reduce the in-plane mismatch (the nominal in-plane lattice parameters of $\mathrm{Cr}$ and BTO are $0.2885 \mathrm{~nm}$ and $0.3992 \mathrm{~nm}$, leading to $2.2 \%$ mismatch for $45^{\circ}$ rotation and $27.7 \%$ for cube-on-cube orientation). Finally, note that the XPD quality is quite high, with peaks clearly emerging from the background, even if the nominal $\mathrm{Cr}$ thickness is very low $(1 \mathrm{~nm})$.

Electron-diffraction patterns from low-energy electron diffraction (LEED) and reflection high-energy electron diffraction (RHEED) appear in the $773 \mathrm{~K}$ annealed film, as shown in Fig. 5, whereas they were not detectable after the annealing at $673 \mathrm{~K}$. This further confirms that the structural order improves as the amorphous $\mathrm{Cr}$ metallic component gets oxidized. The oxidation of $\mathrm{Cr}$ and the crystallization of $\mathrm{CrOx}$ occur concurrently, with both processes originating at the $\mathrm{Cr} / \mathrm{BTO}$ interface, and ordering of the film is achieved alongside oxidation.

Figure 5(a) shows the LEED pattern of BTO at an electron energy of $142 \mathrm{eV}$. The white arrow indicates the [100] direction of the STO substrate. The LEED taken on CrOx at the same energy [Fig. 5(b)] clearly shows the same fourfold symmetry.

The RHEED patterns of CrOx collected with $30 \mathrm{keV}$ electrons on the [100] and [110] azimuths of STO, shown in Figs. 5(d) and 5(f), respectively, confirm the epitaxial relation between the CrOx and BTO. The modulation of the intensity of the streaks that can be observed indicates a three-dimensional (3D) morphology of $\mathrm{CrOx}$ that can be related to surface roughening promoted by the annealing at 673 and $773 \mathrm{~K}$.

The combination of XPD and electron diffraction indicates that $\mathrm{CrOx}$ presents a rocksaltlike structure in which both $\mathrm{Cr}$ and $\mathrm{O}$ atoms share the same coordination. Indeed, in order to account for the +3 oxidation state, $1 / 3$ of the $\mathrm{Cr}$ sites must be vacant, as in the case of defective rocksalt $\mathrm{Cr}$ (III) oxide epitaxially grown by evaporating metallic $\mathrm{Cr}$ on $\mathrm{MgO}$ in an ozone atmosphere [45]. Note that this structure is quite uncommon for $\mathrm{Cr}_{2} \mathrm{O}_{3}$, which typically crystallizes in the 


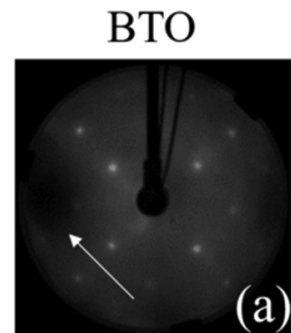

\section{$\mathrm{CrOx} / \mathrm{BTO}$}
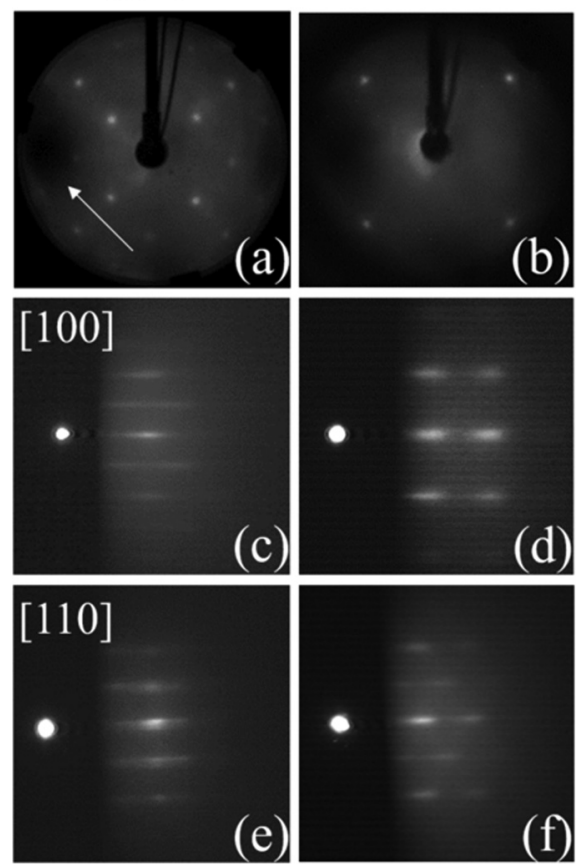

FIG. 5. (a) LEED pattern at $142 \mathrm{eV}$; the white arrow indicates the [100] direction of the STO substrate. (b) LEED pattern at $142 \mathrm{eV}$ of CrOx. (c),(d) RHEED patterns, taken over the [100] azimuth of the STO substrate, of BTO and CrOx, respectively. (e),(f) RHEED patterns, taken over the [110] azimuth of the STO substrate, of BTO and $\mathrm{CrOx}$, respectively.

corundum $\alpha-\mathrm{Cr}_{2} \mathrm{O}_{3}$ or in the defective spinel $\gamma-\mathrm{Cr}_{2} \mathrm{O}_{3}$ phases. In our case, the $\alpha$ phase can be excluded being hcp [46], whereas the $\gamma$ phase would imply a supercell structure that is not shown by electron diffractions [47].

Advanced structural and chemical characterization have been performed by high-resolution (HR)-TEM and STEMEELS, respectively, on an oxidized $\mathrm{Cr}$ film, annealed at $773 \mathrm{~K}$, with $2 \mathrm{~nm}$ nominal Cr thickness (Fig. 6). Figure 6(b) shows a HR-TEM image of the CrOx/BTO interface. A Pt capping layer-deposited prior to sample preparation to protect the $\mathrm{CrOx}$ film-is observed above the $\mathrm{CrOx}$ layer. Figure 6(b) verifies the epitaxial relation between the synthesized oxide and the BTO suggested by diffraction. A cubic structure compatible with the one proposed is found, with CrOx[100]//BTO[100] and $\mathrm{CrOx}[001] / / \mathrm{BTO}[001]$. Extraction of the lattice parameters of $\mathrm{CrOx}$ from TEM gives an in-plane parameter $a=3.99 \pm 0.03 \AA$ and an out-of-plane parameter $c=4.09 \pm 0.04 \AA$, considering the conventional rocksalt cell (the error in extracting lattice parameters with HR-TEM was determined by taking the standard deviation from multiple images and measurements). This corresponds to a small inplane compression $(c / a=1.02)$, as qualitatively predicted by XPD too (see Supplemental Material, Sec. S1 [44]). Note that this growth mode guarantees the lattice matching between CrOx and BTO, with a $0.05 \% \pm 0.86 \%$ in-plane mismatch.

It is worthwhile to note that other $\mathrm{Cr}$ oxides with cubic or tetragonal structure could be potentially grown epitaxially on BTO. CrO, for instance, presents a perfect rocksalt structure with conventional cell size $a=4.16 \AA$ [48] (lattice mismatch with BTO 4\%). Besides being a very unstable compound and difficult to grow in thin films [41], it should be definitely excluded in our case since we observe $\mathrm{Cr}$ in the $3+$ oxidation state. The tetragonal $\mathrm{CrO}_{2}$ with an in plane parameter $a=$ $4.421 \AA$ [48] can be excluded for the same reason as well. Finally, defective spinel $\gamma-\mathrm{Cr}_{2} \mathrm{O}_{3}$, which is isomorphous to $\gamma-\mathrm{Fe}_{2} \mathrm{O}_{3}(a=8.330 \AA$ [48]), could grow on a $2 \times 2$ BTO

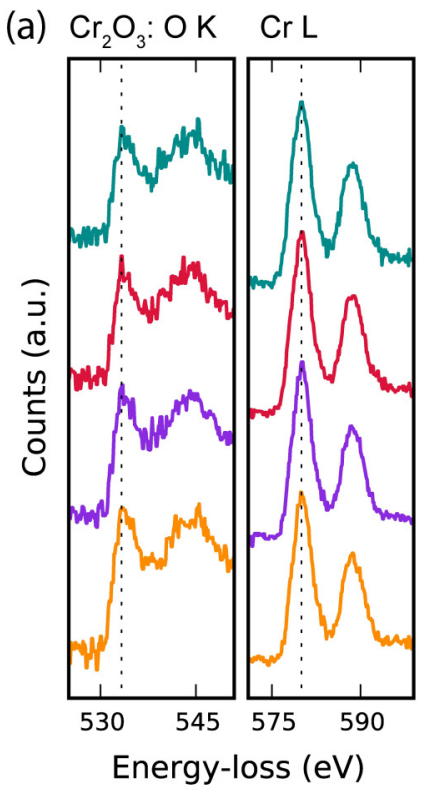

(b)

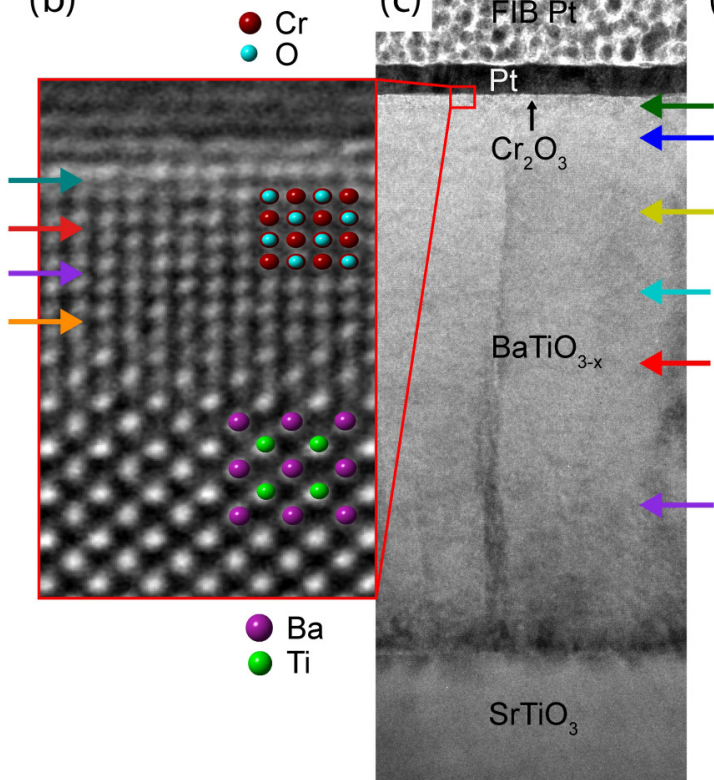

(d)

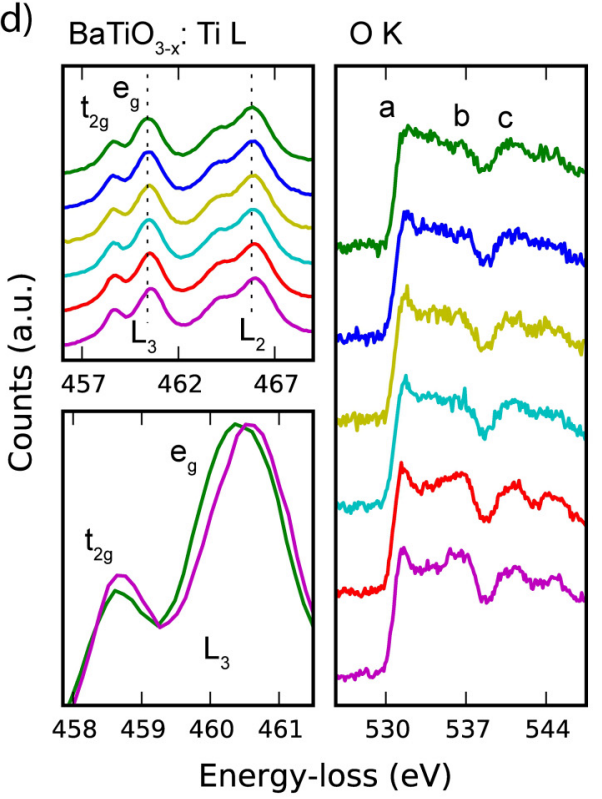

FIG. 6. (a) STEM-EELS spectra at the $\mathrm{O} K$ and $\mathrm{Cr} L$ edges within the CrOx film. (b) Fourier-filtered HR-TEM image of the CrOx/BTO interface showing epitaxial relations between the layers. (c) Low-magnification TEM image of the sample. (d) STEM-EELS spectra at the Ti $L$ and $\mathrm{O} K$ edges within the BTO layer. 


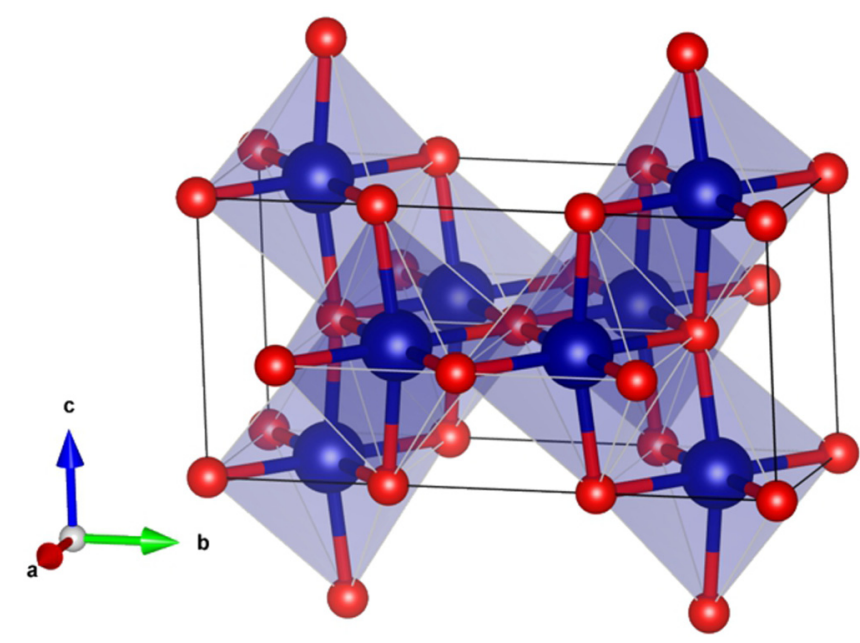

FIG. 7. Crystal structure of the defective rocksalt $\mathrm{Cr}_{2} \mathrm{O}_{3}$ after atomic relaxation. $\mathrm{Cr}$ and $\mathrm{O}$ atoms are shown as blue and red balls, respectively. $\mathrm{CrO}_{6}$ octahedra are shown in light blue.

supercell with $4 \%$ lattice mismatch. This supercell structure, however, is not shown by electron diffractions of CrOx. Finally, we note that the defective rocksalt phase can also grow lattice matched on $\mathrm{MgO}$ substrates $(a=4.217 \AA)$. In that case, the unrelaxed film would have a tensile strain and the out-of-plane parameter would result in $c=3.892 \AA$ [45].

\section{Density functional simulations}

To understand the impact of the defective rocksalt structure on the electronic properties of $\mathrm{CrOx} / \mathrm{BTO}$, DFT calculations have been performed using the information inferred from XPD and TEM. The unit cell used is composed of six $\mathrm{O}$ and four $\mathrm{Cr}$ atoms, as suggested in the literature [45]. Starting from the atomic positions of the perfect rocksalt structure and performing ab initio atomic relaxations, we find a strong displacement of the oxygen atoms. The charged $\mathrm{O}$ atoms tend to move away from the $\mathrm{Cr}$ vacancies for electrostatic reasons. The final crystal configuration of the defective rocksalt is reported in Fig. 7 (see Sec. S2 of the Supplemental Material for the crystal structure model file [44]). Differently from the perfect rocksalt structure, we observe $\mathrm{CrO}_{6}$ octahedra with nonequivalent $\mathrm{Cr}-\mathrm{O}$ bonds and bond angles different from $90^{\circ}$.

The nonequivalent $\mathrm{Cr}-\mathrm{O}$ bonds in-plane are $1.948 \AA$ and $1.953 \AA$, whereas the $\mathrm{Cr}$-O distance out-of-plane is $2.054 \AA$. These values are comparable with the corundum $\mathrm{Cr}_{2} \mathrm{O}_{3}$ phase, where the $\mathrm{Cr}$-O distances are $1.965 \AA$ and $2.016 \AA$ [49]. The inplane $\mathrm{Cr}-\mathrm{O}-\mathrm{Cr}$ bond angles are $87.3^{\circ}, 92.7^{\circ}$, and $93.0^{\circ}$, whereas the out-of-plane $\mathrm{Cr}-\mathrm{O}-\mathrm{Cr}$ bond angle is $93.8^{\circ}$. The in-plane Cr-Cr distances are $2.70 \AA$ and $2.83 \AA$, whereas it is $2.92 \AA$ for the out-of-plane distances. In the corundum $\mathrm{Cr}_{2} \mathrm{O}_{3}$ phase, the Cr-Cr distances are $2.65 \AA$ and $2.89 \AA$, as well comparable to our optimized values in the defective structure.

If we consider the space group Immm, the tetragonal phase strongly differs from the corundum one; however, in both cases, the local coordination is similar: $\mathrm{Cr}$ is surrounded by six oxygens forming a $\mathrm{CrO}_{6}$ octahedron with similar $\mathrm{Cr}-\mathrm{O}$ distances. Therefore, the physical behavior of the $\mathrm{Cr}$ states at the Fermi level is expected to be similar. Furthermore, in both cases, we have distorted octahedra with a crystal field splitting the $d$ manifold into $t_{2 \mathrm{~g}}$ and the $e_{g}$ states.

The $\mathrm{Cr}$ defect reduces the connectivity of the $\mathrm{Cr}$ sublattice, which is in turn expected to decrease the Cr bandwidth, favoring the antiferromagnetic insulating states, as in other transition-metal compounds [50-52]. Indeed, the number of first neighbors in the $\mathrm{Cr}$ sublattice is 12 for the perfect rocksalt and becomes seven in the defective rocksalt. Due to the randomness of the $\mathrm{Cr}$ holes in the structure, we can assume that every $\mathrm{Cr}$ atom has seven first neighbors on average. The connectivity is one of the main differences between the tetragonal and corundum structure. Indeed, the number of first neighbors in the $\mathrm{Cr}$ sublattice is five for the corundum phase and seven for the tetragonal phase of the $\mathrm{Cr}_{2} \mathrm{O}_{3}$. The octahedra are corner shared in the tetragonal phase, whereas face-shared and edge-shared octahedra are present in the corundum phase.

In Fig. 8(a), we show the density of states (DOS) for the defective rocksalt and the corundum phase using $U=0$ and $6 \mathrm{eV}$. In both cases, the system shows an insulating behavior and the low-energy region is dominated by the $3 d$ Cr electrons. The defective rocksalt phase has a smaller energy gap due to the reduced number of nearest neighbors in the $\mathrm{Cr}$ sublattice. As expected, there are three electrons on every $\mathrm{Cr}$ atom that occupy the $t_{2 \mathrm{~g}}$ majority spin states.

With $U=0$, the system presents a $0.8 \mathrm{eV}$ energy gap for the defective rocksalt phase and a $1.0 \mathrm{eV}$ energy gap for the corundum phase [see Fig 8(a), top panel]. As can be seen from Fig. 8(b), the oxygen states are mainly located between -7.5 and $-2.5 \mathrm{eV}$ from the Fermi level. The occupied majority $\mathrm{Cr}$ electrons [Cr up in Fig. 8(b)] lie between $-1.5 \mathrm{eV}$ and the Fermi level, whereas the unoccupied majority electrons are located between 0.8 and $3.0 \mathrm{eV}$. The minority electrons [Cr down in Fig. 8(b)] are predominantly located between 1.5 and $4.5 \mathrm{eV}$.

Upon increasing $U$, the size of the gap increases: for $U=0$, 3 , and $6 \mathrm{eV}$, the gap is $0.8,2.1$, and $3.2 \mathrm{eV}$, respectively, for the defective rocksalt. On the other hand, the bandwidth is barely affected. The band gap of the defective rocksalt is always lower than the gap of the corundum phase, which in the case of $U=6 \mathrm{eV}$ is $3.7 \mathrm{eV}$.

As we can see from the bottom panel of Fig. 8(a), at $U=6 \mathrm{eV}$, the increase of the gap pushes the occupied $d$ states towards the oxygen states, therefore increasing the hybridization between $d$ and $p$ states. In this case, the oxygen states are between -6.5 and $-1.5 \mathrm{eV}$. The occupied majority $\mathrm{Cr}$ electrons lie mostly between $-2.0 \mathrm{eV}$ and the Fermi level, whereas the unoccupied majority electrons are between 3.2 and $5.0 \mathrm{eV}$. The minority electrons are mainly located between 4.0 and $7.0 \mathrm{eV}$.

As discussed in Sec. III A2, XAS spectra are very similar to those of corundum $\alpha-\mathrm{Cr}_{2} \mathrm{O}_{3}$, indicating a strong analogy in the electronic structure of the two systems. This can be indeed related to the similar octahedral coordination of $\mathrm{Cr}$ with oxygen atoms, leading to similar crystal-field splitting of the $d$ states. The $\mathrm{O} K$ XAS allows for a direct comparison between the calculated electronic structure and experimental data, since multiplet effects are not observed in the absorption spectra of ligands in transition metals [42]. From the DFT, we obtain that the first peak of the O $K$ XAS indeed corresponds to the $3 d$ contribution to the DOS, highlighted by an arrow on 

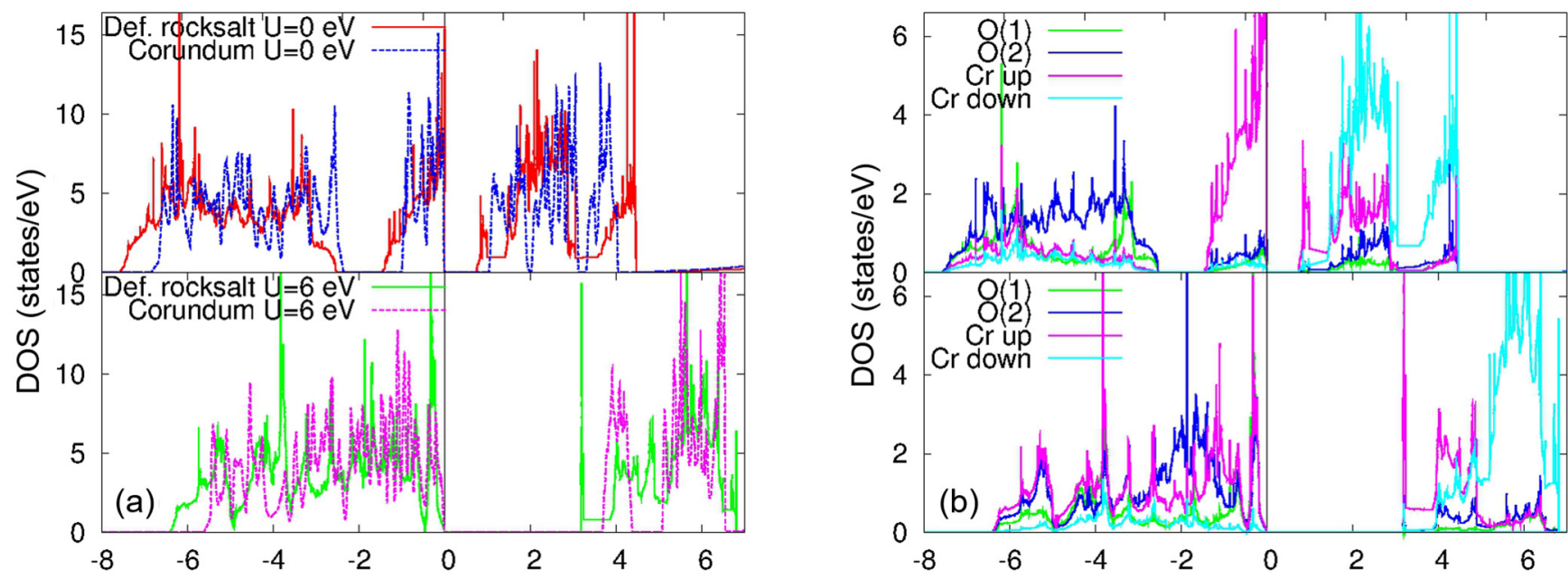

FIG. 8. (a) Total electronic DOS for the defective rocksalt and corundum $\mathrm{Cr}_{2} \mathrm{O}_{3}$ at $U=0 \mathrm{eV}$ (top panel) and $6 \mathrm{eV}$ (bottom panel). (b) Electronic DOS projected on $\mathrm{O}(1), \mathrm{O}(2) \mathrm{Cr}$ up, and $\mathrm{Cr}$ down states for the defective rocksalt $\mathrm{Cr}_{2} \mathrm{O}_{3}$ at $U=0 \mathrm{eV}$ (top panel) and $6 \mathrm{eV}$ (bottom panel). The Fermi level is set to zero.

the first peak of Fig. 3(b). On the contrary, for the prediction of $\mathrm{Cr}-L$ XAS, a ligand field multiplet theory should be used so that we cannot directly compare it with the DFT-based DOS. We recall that following dipole selection rules, O $1 s$ core electrons can be excited only to $p$ states and it has been shown that a good correspondence can be found between the oxygen $p$ states calculated within DFT and the oxygen $K$ absorption spectra. Indeed, when the simulated oxygen $p$ projected DOS for $\mathrm{Cr}_{2} \mathrm{O}_{3}$ empty states is reported in Fig. 3(b), a clear correspondence between the $\mathrm{O} K \mathrm{XAS}$ and the predicted empty states is found.

\section{Oxidation state depth profiling}

Coming back to chemical properties, we consider STEMEELS spectra at the $\mathrm{O} K$ and $\mathrm{Cr} L$ edges [Fig. 6(a)] within the CrOx film and the Ti $L$ and $\mathrm{O} K$ edges within the BTO underlayer [Fig. 6(d)] in order to give a deeper insight on the oxidation process that leads the $\mathrm{Cr}$ layer to become a CrOx film.

In Fig. 6(a), we report the EELS spectra of $\mathrm{O} K$ and $\mathrm{Cr} L$ edges in the CrOx layer, taken at $5 \AA$ step sizes through the film using a probe size of $2 \AA$. The recorded zero loss peak (ZLP) full width at half maximum (FWHM), an estimation of the energy resolution, was measured to be $1.25 \mathrm{eV}$. No peak shift in either edge is observed, indicating that the oxidation state within the CrOx film is uniform. A straightforward method to extract the Cr oxidation state by EELS is to measure the energy difference between $\mathrm{O} \mathrm{K}$ and $\mathrm{Cr} L_{3}$ edges, which acts as a measure of the binding energy difference between the $\mathrm{Cr}$ $2 p_{3 / 2}$ and $\mathrm{O} 1 s$ core levels [53]. This difference, extracted using the edge-onset method [54], is $46.5 \mathrm{eV}$, which is consistent with a +3 oxidation state, as already predicted by both XPS and XAS.

In Fig. 6(d), we present EELS spectra of the $\mathrm{O} K$ and Ti $L$ edges in the BTO layer. For this measurement, the ZLP FWHM was $0.8 \mathrm{eV}$. To correct for drift of the microscopes high-voltage supply, spectra were aligned based on the $\mathrm{Ba} M$ edge. A clear trend is observed in the $\mathrm{O} K$-edge fine structure when moving from the $\mathrm{CrOx} / \mathrm{BTO}$ interface towards the BTO/STO interface.
The $\mathrm{O} K$ prepeak, labeled "a" in Fig. 6(d), is barely observed at the $\mathrm{CrOx} / \mathrm{BTO}$ interface; however, this feature continuously grows in strength when moving towards the STO. The absence of the $\mathrm{O} K$ prepeak in $\mathrm{BTO}$ is associated with oxygen vacancies $[55,56]$. With the presence of oxygen vacancies, a shift from $\mathrm{Ti}^{4+}$ to mixed $\mathrm{Ti}^{4+} / \mathrm{Ti}^{3+}$ is expected in order to maintain charge neutrality ( $\mathrm{Ba}$ is stable at +2 having the inert electronic configuration of noble gas $\mathrm{Xe}$ ). Looking at the Ti $L$ edge, two trends are observed when moving from the CrOx/BTO interface to the BTO/STO interface. First, the Ti $L_{2}$ and $L_{3}$ peaks shift to higher energy (by about $\sim 0.2 \mathrm{eV}$ ), and second, the $t_{2 \mathrm{~g}}$ peaks become more pronounced. Both observations indicate that the Ti within the BTO is partially reduced at the CrOx interface, and that the oxidation state increases towards the nominal value of +4 when moving towards the STO [57]. The spatially modulated $\mathrm{O}$ vacancy concentration and $\mathrm{Ti}$ oxidation state support the hypothesis that the oxidation of the $\mathrm{Cr}$ film is the result of thermally activated diffusion of $\mathrm{O}$ from the underlying BTO film. Note that by comparison with the literature [58,59], we found than even near the CrOx interface, $\mathrm{Ti}$ is much closer to +4 than +3 , indicating only a moderate deoxygenation of the BTO layer, which could be relevant in order to preserve its ferroelectric properties.

\section{CONCLUSIONS}

In this work, we discuss the chemical and structural properties of Cr thin films (1-2 nm thickness) grown by MBE on a $\mathrm{BaTiO}_{3} / \mathrm{SrTiO}_{3}$ (100) template. In situ postannealing was performed at temperatures ranging from 473 to $773 \mathrm{~K}$. A disordered metallic layer was detected for annealing temperatures up to $573 \mathrm{~K}$, whereas at higher temperatures, we observed a progressive oxidation of the chromium layer, which we related to the thermally activated migration of oxygen from the substrate. From XPS, XAS, and EELS, a +3 chromium oxidation state was found. XPD, electron diffractions, and TEM showed a defective rocksalt structure, with one out of every three atoms of chromium missing in order to preserve the stoichiometry $\left(\mathrm{Cr}_{2} \mathrm{O}_{3}\right)$. The structure grows lattice matched on 
the underlying $\mathrm{BaTiO}_{3}$ layer. Despite the structural difference with respect to the corundum $\alpha-\mathrm{Cr}_{2} \mathrm{O}_{3}$ phase, we demonstrated both experimentally (XAS) and theoretically (DFT) that the electronic properties are basically equivalent. This can be ascribed to the similar octahedral coordination of oxygen atoms, leading to similar crystal-field splitting of the $d$ states in the two configurations. Magnetic measurements are in progress in order to confirm the antiferromagnetic character of the film, as predicted by DFT calculations.

\section{ACKNOWLEDGMENTS}

We thank R. Bertacco for fruitful discussion and S. Bertoli for supporting sample growth and characterization. This work was partially funded by Fondazione Cariplo via the project Magister (Project No. 2013-0726). This work was partially performed at Polifab, the micro and nanofabrication facility of Politecnico di Milano, and at the NFFA/APE beamline facility in Trieste, in the framework of the Progetto Internazionale Nanoscience Foundry and Fine Analysis of MIUR, Italy.
[1] G. Radaelli et al., Electric control of magnetism at the $\mathrm{Fe} / \mathrm{BaTiO}_{3}$ interface, Nat. Commun. 5, 3404 (2014).

[2] A. Paul et al., Exotic exchange bias at epitaxial ferroelectricferromagnetic interfaces, Appl. Phys. Lett. 105, 022409 (2014).

[3] S. Hausmann et al., Atomic-scale engineering of ferroelectricferromagnetic interfaces of epitaxial perovskite films for functional properties, Sci. Rep. 7, 10734 (2017).

[4] C.-G. Duan, S. S. Jaswal, and E. Y. Tsymbal, Predicted Magnetoelectric Effect in $\mathrm{Fe} / \mathrm{BaTiO}_{3}$ Multilayers: Ferroelectric Control of Magnetism, Phys. Rev. Lett. 97, 047201 (2006).

[5] M. Asa, L. Baldrati, C. Rinaldi, S. Bertoli, G. Radaelli, M. Cantoni, and R. Bertacco, Electric field control of magnetic properties and electron transport in $\mathrm{BaTiO}_{3}$-based multiferroic heterostructures, J. Phys.: Condens. Matter 27, 504004 (2015).

[6] W. Eerenstein, M. Wiora, J. L. Prieto, J. F. Scott and N. D. Matur, Giant sharp and persistent converse magnetoelectric effects in multiferroic epitaxial heterostructures, Nat. Mater. 6, 348 (2007).

[7] G. Radaelli, D. Gutiérrez, F. Sanchéz, R. Bertacco, M. Stengel, and J. Fontcuberta, Large room-temperature electroresistance in dual-modulated ferroelectric tunnel barriers, Adv. Mater. 27, 2602 (2015)

[8] D. J. Kim, H. Lu, S. Ryu, C.-W. Bark, C.-B. Eom, E. Y. Tsymbal, and A. Gruverman, Ferroelectric tunnel memristor, Nano Lett. 12, 5697 (2012).

[9] A. K. Bain and P. Chand, Ferroelectrics (Wiley-VCH, New York, 2017).

[10] G. B. Haxel, J. B. Hedrick, and G. J. Orris, Rare Earth Elements-Critical Resources for High Technology, U.S. Geological Survey, 087-02 (2005), http://pubs.usgs.gov/fs/ 2002/fs087-02.

[11] E. Fawcett, Spin-density-wave antiferromagnetism in chromium, Rev. Mod. Phys. 60, 209 (1988).

[12] M. C. Hanf, C. Pirri, J. C. Peruchetti, D. Bolmont, and G. Gewinner. Formation of an interfacial alloy and epitaxial bcc Cr layers on Au(100), Phys. Rev. B 39, 1546 (1989).

[13] C. Krembel, M. C. Hanf, J. C. Peruchetti, D. Bolmont, and G. Gewinner, Growth of a flat Cr monolayer on $\operatorname{Ag}(100)$, Phys. Rev. B 44, 8407(R) (1991).

[14] M. Riva, A. Picone, G. Bussetti, A. Brambilla, A. Calloni, G. Berti, L. Duò, F. Ciccacci, and M. Finazzi, Oxidation effects on ultrathin $\mathrm{Ni}$ and $\mathrm{Cr}$ films grown on $\mathrm{Fe}(001)$ : A combined scanning tunneling microscopy and Auger electron spectroscopy study, Surf. Sci. 621, 55 (2014).

[15] M. A. Leroy et al., Tunnel-mediated coupling between antiferromagnetic thin films, Phys. Rev. B 90, 035432 (2014).
[16] Q. Fu and T. Wagner, Nucleation and growth of Cr clusters and films on (100) $\mathrm{SrTiO}_{3}$ surfaces, Thin Solid Films 420-421, 455 (2002).

[17] Q. Fu and T. Wagner, Metal/oxide interfacial reactions: Oxidation of metals on $\mathrm{SrTiO}_{3}(100)$ and $\mathrm{TiO}_{2}$ (110), J. Phys. Chem. B 109, 11697 (2005).

[18] T. Wagner, Q. Fu, C. Winde, S. Tsukimoto, and F. Phillipp, A comparative study of the growth of $\mathrm{Cr}$ on $(110) \mathrm{TiO}_{2}$ rutile, (0001) $\alpha-\mathrm{Al}_{2} \mathrm{O}_{3}$ and (100) $\mathrm{SrTiO}_{3}$ surfaces, Interface Sci. 12, 117 (2004).

[19] Q. Fu and T. Wagner, Thermal stability of $\mathrm{Cr}$ clusters on $\mathrm{SrTiO}_{3}(100)$, Surf. Sci. 505, 39 (2002).

[20] Q. Fu and T. Wagner, On the tunability of chemical reactions at metal-oxide interfaces, Surf. Sci. 574, L29 (2005).

[21] R. Bertacco, M. Cantoni, M. Riva, A. Tagliaferri, and F. Ciccacci, Epitaxial growth and characterization of layered magnetic nanostructures, Appl. Surf. Sci. 252, 1754 (2005).

[22] G. Radaelli, S. Brivio, I. Fina, and R. Bertacco, Correlation between growth dynamics and dielectric properties of epitaxial $\mathrm{BaTiO}_{3}$ films, Appl. Phys. Lett. 100, 102904 (2012).

[23] S. Balaz, Z. Zeng, and L. J. Brillson, Heterojunction band offsets and dipole formation at $\mathrm{BaTiO}_{3} / \mathrm{SrTiO}_{3}$ interfaces, J. Appl. Phys. 114, 183701 (2013).

[24] S. Nayak, B. Sahoo, K. Chaki, and D. Khastgir, Facile preparation of uniform barium titanate $\left(\mathrm{BaTiO}_{3}\right)$ multipods with high permittivity: Impedance and temperature dependent dielectric behavior, RSC Adv. 4, 1212 (2014).

[25] G. Panaccione et al., Advanced photoelectric effect experiment beamline at Elettra: A surface science laboratory coupled with Synchrotron Radiation, Rev. Sci. Instrum. 80, 043105 (2009).

[26] J. L. Hart, A. C. Lang, A. C. Leff, P. Longo, C. Trevor, R. D. Twesten, and M. L. Taheri, Direct detection electron energy-loss spectroscopy: A method to push the limits of resolution and sensitivity, Sci. Rep. 7, 8243 (2017).

[27] G. Kresse and D. Joubert, From ultrasoft pseudopotentials to the projector augmented-wave method, Phys. Rev. B 59, 1758 (1999).

[28] J. P. Perdew, A. Ruzsinszky, G. I. Csonka, O. A. Vydrov, G. E. Scuseria, L. A. Constantin, X. Zhou, and K. BurkePhys. Restoring the density-gradient expansion for exchange in solids and surfaces, Rev. Lett. 100, 136406 (2008).

[29] V. I. Anisimov, J. Zaanen, and O. K. Andersen, Band theory and Mott insulators: Hubbard $U$ instead of Stoner I, Phys. Rev. B 44, 943 (1991). 
[30] A. I. Liechtenstein, V. I. Anisimov, and J. Zaanen, Densityfunctional theory and strong interactions: Orbital ordering in Mott-Hubbard insulators, Phys. Rev. B 52, 5467(R) (1995).

[31] P. E. Blöchl, Projector augmented-wave method, Phys. Rev. B 50, 17953 (1994).

[32] J. D. Pack and H. J. Monkhorst, Special points for Brillouin-zone integrations-A reply, Phys. Rev. B 16, 1748 (1977).

[33] G. Radaelli, M. Cantoni, L. Lijun, M. Espahbodi, and R. Bertacco, Two dimensional growth of ultrathin Fe films on $\mathrm{BaTiO}_{3}$ with sharp chemical interface, J. Appl. Phys. 115, 063501 (2014).

[34] S. Tanuma, C. J. Powell, and D. R. Penn, Calculations of electron inelastic mean free paths. II. Data for 27 elements over the 50$2000 \mathrm{eV}$ range, Surf. Interface Anal. 17, 911 (1991).

[35] M. C. Biesinger, C. Brown, J. R. Mycroft, R. D. Davidson, and N. S. McIntyre, X-ray photoelectron spectroscopy studies of chromium compounds, Surf. Interface Anal. 36, 1550 (2004).

[36] J. F. Moulder, W. F. Stickle, P. E. Sobol, K. D. Bomben, Handbook of X-ray Photoelectron Spectroscopy, edited by J. Chastain and R. C. King, Jr. (Perkin-Elmer Corporation, Physical Electronics Division, Minnesota, 1995).

[37] B. Chan, A. Karton, K. Raghavacari, and L. Radom, Heats of formation for $\mathrm{CrO}, \mathrm{CrO}_{2}$, and $\mathrm{CrO}_{3}$ : An extreme challenge for blackbox composite procedures, J. Chem. Theory Comput. 8, 3159 (2012).

[38] C. Theil, J. van Elp, and F. Folkmann, Ligand field parameters obtained from and chemical shifts observed at the $\mathrm{Cr} L_{2,3}$ edges, Phys. Rev. B 59, 7931 (1999).

[39] Yu. S. Dedkov et al., Correlations in the electronic structure of half-metallic ferromagnetic $\mathrm{CrO}_{2}$ films: An x-ray absorption and resonant photoemission spectroscopy study, Phys. Rev. B 72, 060401(R) (2005).

[40] S. O. Kucheyeva, B. Sadigh, T. F. Baumann, Y. M. Wang, and T. E. Felter, Electronic structure of chromia aerogels from soft Xray absorption spectroscopy, J. Appl. Phys. 101, 124315 (2007).

[41] O. C. Rogojanu, G. A. Sawatzky, and L. H. Tjeng, Stabilizing $\mathrm{CrO}$ by epitaxial growth, Ph.D. thesis, University of Groningen, 2002.

[42] F. de Groot and A. Kotani, Core Level Spectroscopy of Solids (CRC Press, Boca Raton, 2008).

[43] F. M. F. De Groot, M. Grioni, J. C. Fuggle, J. Ghijsen, G. A. Sawatzky, and H. Petersen, Oxygen 1s x-ray-absorption edges of transition-metal oxides, Phys. Rev. B 40, 5715 (1989).

[44] See Supplemental Material at http://link.aps.org/supplemental/ 10.1103/PhysRevMaterials.2.033401 for the diffraction angles in XPD from $\mathrm{CrOx} / \mathrm{BTO}$ and the structure model file of the defective rocksalt structure.

[45] X. S. Du, S. Hak, T. Hibma, O. C. Rogojanu, and B. Struth, $\mathrm{X}$-rays diffraction on a new chromium oxide single-crystal thin film prepared by molecular beam epitaxy, J. Cryst. Growth 293, 228 (2006).

[46] M. Lubbe and W. Moritz, A LEED analysis of the clean surfaces of $\alpha-\mathrm{Fe}_{2} \mathrm{O}_{3}(0001)$ and $\alpha-\mathrm{Cr}_{2} \mathrm{O}_{3}(0001)$ bulk single crystals, J. Phys.: Condens. Matter 21, 134010 (2009).

[47] Y. Gao and S. A. Chambers, Heteroepitaxial growth of $\alpha-\mathrm{Fe}_{2} \mathrm{O}_{3}, \gamma-\mathrm{Fe}_{2} \mathrm{O}_{3}$ and $\mathrm{Fe}_{3} \mathrm{O}_{4}$ thin films by oxygen-plasmaassisted molecular beam epitaxy, J. Cryst. Growth 174, 446 (1997).

[48] S. Grazulis, D. Chateigner, R. T. Downs, A. T. Yokochi, M. Quiros, L. Lutterotti, E. Manakova, J. Butkus, P. Moeck, and A. Le Bail, Crystallography open database - An openaccess collection of crystal structures, J. Appl. Cryst. 42, 726 (2009).

[49] G. K. Lewis and H. G. Drickamer, Effect of high pressure on the lattice parameters of $\mathrm{Cr}_{2} \mathrm{O}_{3}$ and $\alpha-\mathrm{Fe}_{2} \mathrm{O}_{3}$, J. Chem. Phys. 45, 224 (1966).

[50] C. Autieri, Antiferromagnetic and xy ferro-orbital order in insulating $\mathrm{SrRuO}_{3}$ thin films with $\mathrm{SrO}$ termination, J. Phys. Condens. Matter 28, 426004 (2016).

[51] S. A. Ivanov et al., Polar order and frustrated antiferromagnetism in perovskite $\mathrm{Pb}_{2} \mathrm{MnWO}_{6}$ single crystals, Inorg. Chem. 55, 2791 (2016).

[52] D. J. Groenendijk et al., Spin-Orbit Semimetal $\mathrm{SrIrO}_{3}$ in the Two-Dimensional Limit, Phys. Rev. Lett. 119, 256403 (2017).

[53] A. M. Arevalo-Lopez and M. A. Alario-Franco, Reliable method for determining the oxidation state in chromium oxides, Inorgan. Chem. 48, 11843 (2009).

[54] H. Tan, J. Verbeeck, A. Abakumov, and G. V. Tendeloo, Oxidation state and chemical shift investigation in transition metal oxides by EELS, Ultramicroscopy 116, 24 (2012).

[55] G. Y. Yang, S. I. Lee, Z. J. Lui, C. J. Anthony, E. C. Dickey, Z. K. Lui, and C. A. Randall, Effect of local oxygen activity on $\mathrm{Ni}-\mathrm{BaTiO}_{3}$ interfacial reactions, Acta Mater. 54, 3513 (2006).

[56] R. Jie and Z. Jing, EELS study on BST thin film under electron beam irradiation, Sci. China Ser. E: Technolog. Sci. 47, 659 (2004).

[57] A. Ohtomo, D.A. Muller, J. L. Grazul, and H. Y. Hwang, Artificial charge-modulation in atomic-scale perovskite titanate superlattices, Nature (London) 419, 378 (2002).

[58] Y. Shao, C. Maunders, D. Rossouw, T. Kolodiazhnyi, and G. A. Botton, Quantification of the Ti oxidation state in $\mathrm{BaTi}_{1-x} \mathrm{Nb}_{x} \mathrm{O}_{3}$ compounds, Ultramicroscopy 110, 1014 (2010).

[59] M.-G. Han, M.S.J. Marshall, L. Wu, M. A. Schofield, T. Aoki, R. Twesten, J. Hoffman, F.J. Walker, c.-H. Han, and Y. Zhu, Interface-induced nonswitchable domains in ferroelectric thin films, Nat. Commun. 5, 4693 (2014). 